\title{
Las medidas no farmacológicas en el manejo de la Hipertensión Arterial
}

\author{
Santiago Reinoso Hermida ${ }^{a}$, Gabriel J. Díaz Grávalos ${ }^{b}$
}

${ }^{\text {a }}$ MIR de $3^{\circ}$ año de Medicina de Familia y Comunitaria (Unidad Docente de Ourense). Centro de Saúde O Carballiño. O Carballiño (Ourense).

b Medicina de Familia. Centro de Saúde Cea. Cea (Ourense).

Correspondencia:

Gabriel J. Díaz Grávalos,

C/ Feria n 9, 32130 -

Cea (Ourense, España). E-mail: gdgravalos@hotmail.com.

Recibido el 17 de febrero de 2009.

Aceptado para su publicación el 15 de mayo de 2009.

\section{RESUMEN}

Objetivo. Conocer la utilización por los médicos de familia de las medidas no farmacológicas (MNF) recomendadas en el tratamiento de la hipertensión arterial.

Diseño. Estudio transversal mediante encuesta a pacientes.

Emplazamiento. Consultas de Atención Primaria rurales y urbanas.

Participantes. Pacientes hipertensos pertenecientes a 30 médicos urbanos y rurales.

Mediciones principales. Se registró la prescripción de MNF (reducción de peso y consumo de sal, dieta equilibrada, realización de actividad física, reducción del consumo de alcohol, abandono del tabaco). Si la medida no procedía, se hizo constar así. Los datos fueron analizados con SPSS.15.0. Resultados. Se incluyeron 150 pacientes, 70 (46,7\%) varones, con una edad media de 65,5 años (DE 10,2). El 50\% residía en medio urbano. De las medidas consideradas se recomendaba el $77,5 \%$, recibiendo algún consejo el $98 \%$ de los pacientes. El $42 \%$ recibió consejo sobre la totalidad de las medidas que le correspondían. No hubo diferencias estadísticamente significativas entre médicos urbanos y rurales, ni en función del sexo o la edad del facultativo, siendo más frecuente en los pacientes de menor edad (Rho de Spearman 0,28; $p<0,001$ ). Un análisis de regresión, considerando el grado de realización de consejo como variable dependiente, mostró relación significativa con la edad de los pacientes $(t=-2,9 ; p=0,04)$.

Conclusiones: Es posible incrementar la utilización de medidas no farmacológicas en el tratamiento de la Hipertensión Arterial en nuestro medio. Los pacientes más jóvenes reciben más consejo sobre estas medidas. No hubo diferencias en función del lugar de trabajo, sexo o edad del médico.

Palabras clave. Hipertensión. Estilo de Vida. Educación en Salud.

\section{ABSTRACT}

Non-pharmacological measures in the management of hypertension

Objective. To determine family physicians' (FP) use of the recommended non-pharmacological treatment (NPT) for hypertension.

Design. Cross-sectional survey.

Setting. Primary care practices in rural and urban areas.

Participants. Patients with hypertension from 30 urban and rural practices.

Measurements. We recorded the prescription of NPT (weight loss, alcohol and salt intake reduction, diet, physical activity, and smoking cessation). If the measure was not applicable, it was recorded as such. Data were analyzed with SPSS.15.0.

Results. We included 150 patients, 70 (46.7\%) males. Mean (SD) age was 65.5 (10.2) years, and $50 \%$ resided in urban areas. $77.5 \%$ of the above measures were recommended, $98 \%$ of the patients were receiving some counselling and $42 \%$ received counselling on all the indicated measures. There were no significant differences between urban and rural doctors, or according to the sex or age of the physicians. Counselling was more common in younger patients (Spearman _ - 0.28, p <0001). A regression analysis considering the degree of counselling as the dependent variable showed a significant association of counselling with patient age $(t=-2.9, p=0.04)$.

Conclusions. Non-pharmacological treatment for hypertension could be increased in the Primary Care setting. Older people were less likely to be given relevant counselling. There were no differences as regards FP rural/urban practice, sex or age.

Key words: Hypertension. Life Style. Health Education.

Un resumen de este estudio se presentó como Comunicación en las XIII Jornadas Gallegas de Medicina de Familia y Comunitaria (Vigo, mayo de 2007). 


\section{INTRODUCCIÓN}

Las Guías de Práctica Clínica ${ }^{1,2}$ recomiendan que el planteamiento terapéutico de los pacientes hipertensos considere siempre, bien como único procedimiento, bien como complemento del tratamiento farmacológico, la utilización de diversas medidas no farmacológicas (MNF), las cuales contribuyen a alcanzar y mantener el control de las cifras de presión arterial (PA). Es sabido que incluso pequeños descensos de la PA pueden desembocar en importantes reducciones de la morbimortalidad relacionada con ella ${ }^{3}$.

Un reciente metaanálisis de estudios realizados en países occidentales mostraba la importancia de los factores relacionados con el estilo de vida en la aparición de la $\mathrm{HTA}^{4}$. Es conocido igualmente que la realización de MNF favorece la efectividad de la medicación específica, a la par que consigue disminuir la importancia de otros factores reconocidos de riesgo cardiovascular, directos o indirectos ${ }^{5}$.
Por todo ello, la recomendación de dichas MNF debería ser universal y, dado el escaso éxito que presenta el consejo sobre estilos de vida, debería ser realizada de forma prácticamente continua a lo largo de la relación del Médico de Familia (MF) con el paciente hipertenso ${ }^{1}$.

La escasa efectividad del consejo hace que con alguna frecuencia el MF obvie casi por completo estas recomendaciones ${ }^{6}$, ciñéndose al tratamiento farmacológico. Se han reconocido diversas causas que pueden explicar este hecho ${ }^{7,8}$, tanto en relación con el facultativo como con el paciente o el medio.

Con estas premisas, el objetivo de este estudio es comprobar la frecuencia de recomendación de las MNF para el control de la PA en la práctica real según la opinión de los pacientes, y valorar las posibles diferencias en función de algunas características de los facultativos.
Centro de salud:

Edad:

Sexo:

1 ¿Qué medidas no farmacológicas le prescribió su médico para su HTA?

- Reducir el peso

- Moderar el consumo de alcohol

Realizar actividad física

- Reducir el consumo de sal

Dieta equilibrada (mediterránea)

A Abandono del tabaco

2 ¿Qué medidas no farmacológicas realiza usted para tratar su HTA?

- Reducir el peso

Moderar el consumo de alcohol

Realizar actividad física

Reducir el consumo de sal

Dieta equilibrada (mediterránea)

a Abandono del tabaco

3 Medicación antihipertensiva actual:

$\square$ No

Monoterapia

Biterapia

$\square$ Triterapia

4 ¿Buen control de T.A.? (última medición $\leq 140 / 90$ )

$\square$ Sí

$\square$ No

$\square$ No mide

Tabla 1. Encuesta aplicada a los pacientes. 


\begin{tabular}{lcc}
\hline Medida no farmacológica & Recomendada* $^{*}$ & Cumplimentada* $^{*}$ \\
\hline Reducir peso & $57,4 \%$ & $35,2 \%$ \\
Reducir consumo de alcohol & $85,7 \%$ & $70,1 \%$ \\
Consumir dieta equilibrada & $73,0 \%$ & $50,0 \%$ \\
Aumentar actividad física & $83,1 \%$ & $52,7 \%$ \\
Disminuir consumo de sal & $95,3 \%$ & $87,3 \%$ \\
Dejar de fumar & $48,3 \%$ & $13,8 \%$ \\
\hline
\end{tabular}

Tabla 2. Porcentaje de pacientes a los que se recomendó cada medida y porcentaje de cumplimiento declarado.

*Porcentajes sobre pacientes en los que procedía la aplicación de la medida.

\section{MATERIAL Y MÉTODO}

Se seleccionaron mediante muestreo de conveniencia 30 MF de la provincia de Ourense, la mitad con ejercicio en el medio rural. Una vez obtenida su autorización, se encuestó a 5 pacientes de cada uno de los facultativos seleccionados a la salida de las respectivas consultas. La selección de estos pacientes se realizó de forma aleatoria, previa comprobación de su condición de hipertenso y explicación de la finalidad del cuestionario. Para evitar sesgos motivados por la observación, no se informó a los facultativos seleccionados de la naturaleza exacta de las preguntas a realizar a sus pacientes.

Las encuestas fueron realizadas por dos médicos que acordaron previamente el procedimiento a seguir. La encuesta fue ensayada con 10 pacientes en un centro de salud rural, para valorar posibles dificultades en su comprensión o cumplimentación. Para los efectos de este estudio se consideró medio rural el que presentaba una densidad de población inferior a los 150 habitantes por kilómetro cuadrado (Criterio OCDE).

Las MNF consideradas fueron las referenciadas en las recomendaciones 2007 de la Sociedad Europea de Hipertensión y la Sociedad Europea de Cardiología ${ }^{1}$. La tabla 1 muestra el contenido de la encuesta realizada. Cuando por las características del paciente (no ser fumador, estar incapacitado para el ejercicio físico, no presentar sobrepeso o no consumir alcohol) la medida recomendada no procedía, se hizo constar así. Se consideró que las recomendaciones de reducción del consumo de sal y adaptación de una dieta equilibrada deberían ser aplicadas a todo hipertenso.

El análisis de los datos se realizó con ayuda del programa SPSS versión 15.0. Las variables cuantitativas se describieron como media y desviación estándar (DE) y las cualitativas como porcentajes. Se determinaron los intervalos de confianza del 95\%. Se utilizó en el análisis la prueba t de Student, $\chi^{2}$, correlación y regresión lineal, según fue necesario. En el análisis multivariante fueron incluidas como variables predic- toras edad, sexo y medio de residencia. El grado de significación estadística que se consideró fue un valor de $p \leq 0,05$.

\section{RESULTADOS}

Se incluyeron 150 pacientes, de los que $70(46,7 \%)$ eran varones. La edad media era de 65,5 (DE 10,2) años. El 50\% residía en medio urbano. El 47,3\% de los pacientes afirmó encontrarse en monoterapia, sin diferencias en función del medio de residencia, refiriendo un grado de control adecuado el $64 \%$ de los pacientes.

La tabla 2 muestra los porcentajes de pacientes que afirmaron que se les habían recomendado las diferentes MNF y el grado de cumplimiento referido (porcentajes sobre pacientes en los que procedía la aplicación de dichas medidas). Siempre según la opinión de los pacientes, del total de medidas consideradas se recomendaba el $77,5 \%$, recibiendo algún tipo de consejo el $98 \%$ de los individuos. El $42 \%$ recibió consejo sobre la totalidad de las medidas que le correspondían.

La recomendación sobre consumo de tabaco y alcohol era más frecuente en el varón $(p=0,001)$. El consejo más frecuentemente citado fue la reducción del consumo de sal y el menos citado la supresión del tabaco $(p<0,001)$. No hubo diferencias estadísticamente significativas entre médicos urbanos y rurales, ni en función del sexo o la edad del facultativo, siendo más frecuente la realización de consejo en los pacientes de menor edad (Rho de Spearman 0,28; $p<0,001$ ). Un análisis de regresión lineal, considerando el grado de realización de consejo como variable dependiente, mostró relación significativa con la edad de los pacientes $(t=-2,9 ; p=0,04)$.

\section{DISCUSIÓN}

En general, los MF afirman que las recomendaciones a los pacientes sobre estilo de vida forman parte de su práctica habitual ${ }^{9}$. Sin embargo, estos datos suelen estar basados en respuestas a cuestionarios realizados sobre los propios facultativos, por lo que es 
probable que exista una sobrestimación del consejo realizado. En este estudio la frecuencia de consejo puede considerarse elevada si comparamos con los datos de otros similares. Milder et $\mathrm{al}^{10}$, en una investigación realizada con videograbación de las consultas, encontraron que sólo en el $40 \%$ de las entrevistas con hipertensos se discutían aspectos relacionados con el estilo de vida, cifra similar a la encontrada por Kreuter ${ }^{11}$ con un método similar al utilizado aquí, y muy alejada de los pobres resultados obtenidos en la década de los 90 en análisis realizados por métodos similares al presente ${ }^{12}$. Al respecto, existen estudios que demuestran una buena consistencia entre la realización de consejo por parte del facultativo y el recuerdo del mismo por parte del paciente ${ }^{13}$, por lo que se puede considerar que este tipo de enfoque permite un conocimiento lo suficientemente cercano a la realidad.

Si se desglosan las diferentes recomendaciones consideradas, se aprecia una menor frecuencia de intervención sobre los factores de riesgo de más difícil manejo, como son el sobrepeso o el tabaquismo. En concreto, destaca negativamente la baja frecuencia de consejo antitabáquico, hecho coincidente con lo encontrado por otros autores ${ }^{10,11}$.

Nuestros resultados muestran un peor índice de consejo en el paciente de más edad. Este hallazgo es contradictorio en la literatura ${ }^{10}$, aunque también existen estudios que encuentran diferencias en detrimento de la persona mayor ${ }^{14}$. La consideración de que los cambios en el estilo de vida pueden ser más beneficiosos en el paciente más joven pueden ser una explicación para sustentar este resultado.

Este estudio pretende valorar la frecuencia de consejo sobre estilo de vida en la práctica. Por tanto, no se valoró la pertinencia y adecuación del consejo realizado respecto a las recomendaciones actuales, lo que debería ser objeto de investigación posterior. Los resultados deben ser considerados teniendo en cuenta las limitaciones inherentes a este tipo de encuestas. No se comprobó que las declaraciones de los pacientes sobre la realización de consejo se correspondieran con la realidad, existiendo la posibilidad de que el paciente, como en toda encuesta, afirmara lo que le parecía correcto o falseara la realidad, pero éste es un problema propio de los estudios realizados mediante este sistema. Además, la hipertensión es una condición difundida entre los pacientes y, por tanto, las recomendaciones más habituales pueden ser conocidas aunque realmente no haya existido el consejo.
Por otra parte, la selección no aleatoria de los facultativos también puede sesgar los resultados; sin embargo, dicha selección respondió exclusivamente a criterios de accesibilidad y por tanto no se prejuzgó el estilo de práctica del profesional, lo que, unido al número apreciable de facultativos incluido, creemos que permite ofrecer un cuadro fiable de la realidad.

\section{BIBLIOGRAFÍA}

1. The Task Force for the Management of Arterial Hypertension of the European Society of Hypertension (ESH) and of the European Society of Cardiology (ESC). 2007 Guidelines for the Management of Arterial Hypertension. J Hypert 2007; 25:1005187.

2. Sociedad Española de Hipertensión - Liga Española de Hipertensión Arterial. Guía Española de Hipertensión Arterial 2005. Hipertensión 2005; 22 Supl 2:1-84.

3. Collins R, Peto R, MacMahon S, Hebert P, Fiebach NH, Eberlein $\mathrm{KA}$ et al. Blood pressure, stroke, and coronary heart disease. Part 2, short-term reductions in blood pressure: overview of randomized drug trials in their epidemiological context. Lancet 1990; 335:827-38.

4. Geleijnse JM, Kok FJ, Grobbee DE. Impact of dietary and lifestyle factors on the prevalence of hypertension in Western populations. Eur J Public Health 2004; 14:235-9.

5. Writing Group of the PREMIER Collaborative Research Group. Effects of Comprehensive Lifestyle Modification on Blood Pressure Control: Main Results of the PREMIER Clinical Trial. JAMA 2003; 289:2083-93.

6. Lawlor DA, Keen S, Neal RD. Can general practitioners influence the nation's health through a population approach to provision of lifestyle advice? Br J Gen Pract 2000; 50:455-9.

7. Valente CM, Sobal J, Muncie HL, Levine DV, Antlitz AM. Health promotion: physician's beliefs, attitudes and practices. Am J Prev Med 1982; 2:82-8.

8. Yeager KK, Donehoo RS, Macera CA, Croft JB, Heath GW, Lane MJ. Health promotion practices among physicians. Am J Prev Med 1996; 2:238-41.

9. Frijling BD, Lobo CM, Hulscher MEJL, Van Drenth BB, Braspenning JC, Prins A et al. Provision of information and advice in cardiovascular care: clinical performance of general practitioners. Patient Educ Couns 2002, 2:131-7.

10. Milder IEJ, Blokstra A, de Groot J, van Dulmen S, Bemelmans WJE. Lifestyle counseling in hypertension-related visits - analysis of video-taped general practice visits. BMC Family Practice 2008, 9:58

11. Kreuter MW, Chheda SG, Bull FC. How does physician advice influence patient behaviour? Arch Fam Med 2000; 9:426-33.

12. Silagy C, Muir J, Coulter A, Thorogood M, Yudkin P, Roe L. Lifestyle advice in general practice: rates recalled by patients. BMJ 1992; 305:871-4.

13. Pill RM, Jones-Elwyn G, Stott $\mathrm{NCH}$. Opportunistic health promotion: quantity or quality? J R Coll Gen Pract 1989; 39:196200.

14. Foss FA, Dickinson E, Hills M, Thomson A, Wilson V, Ebrahim S. Missed opportunities for the prevention of cardiovascular disease among British hypertensives in primary care. Br J Gen Pract 1996; 46:571-5. 